M. Asghari-Larimi, P. Corsini, E. Ranjbar-Yanehsari / TJ MCS Vol .5 No. 2 (2012) 115-125

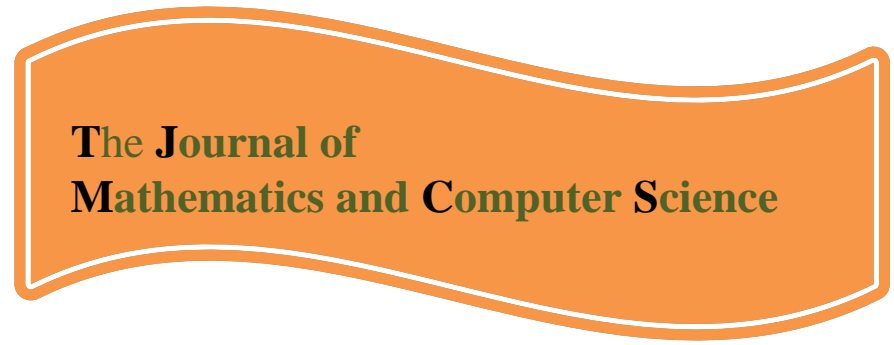

Available online at

http://www.TJMCS.com

The Journal of Mathematics and Computer Science Vol .5 No.2 (2012) 115-125

\title{
Intuitionistic fuzzy sets and join spaces associated with $n$-ary membership functions
}

\author{
Mohsen Asghari-Larimi \\ Department of Mathematics, GolestanUniversity, Gorgan, Iran \\ asghari2004@yahoo.com \\ Piergiulio Corsini \\ Dipartimento di Matematica e Informatica, ViadelleScienze 206, 33100 Udine, Italy \\ corsini@dimi.uniud.it \\ Esmail Ranjbar-Yanehsari \\ Department of Mathematics,Faculty of Sciences, Gorgan Branch,Islamic Azad University,Gorgan, Iran \\ es_ranjbar@yahoo.com
}

Received: February 2012, Revised: November 2012

Online Publication: December 2012

\begin{abstract}
In this paper, we associate finite hyperstructures with fuzzy sets endowed with $n$-ary membership functions and analyze the properties of this new hyperstructures. We prove that the new hyperstructure is a commutative hypergroup, but generally it is not a join space. We give some conditions such that the hypergroup has this property. In particular, we investigate some natural equivalence relations on the set of all intuitionistic fuzzy sub-hypergroups of a hypergroup.
\end{abstract}

Keywords: Hyperstructure; Join space; Fuzzy subhypergroup; Intuitionistic fuzzy subhypergroups.

2010 Mathematics Subject Classification: 20 N20.

\section{Introduction.}

The theory of algebraic hyperstructures, which is a generalization of the theory of ordinary algebraic structures, was first introduced by F. Marty, in [15]. Since then many researchers have studied the theory of hyperstructures and developed the theory from the algebraic point of view. This theory also has many applications in other are as such as in geometry, graphs and hypergraphs, lattices, fuzzy and rough sets, automata, cryptography, codes, etc (see $[1,2,3,4,5,6$, $7,9,16])$. 
M. Asghari-Larimi, P. Corsini, E. Ranjbar-Yanehsari / TJMCS Vol .5 No. 2 (2012) 115-125

L.A. Zadeh, in [19], introduced the notion of a fuzzy subset of a non-empty set $X$, as a function from $X$ to $[0,1]$. This notion has opened an important research field of mathematics in the past decades. P. Corsini initiated an area of research by making a connection between hyperstructures and fuzzy sets: he associated a join space with a fuzzy set, in [8], and then a fuzzy set with a hypergroupoid, in [11].

Another topic in the relation between hypergroups and fuzzy sets was introduced by P. Corsini, in [10]: he constructed a new finite hypergroupoid using two membership functions. He proved that, in general, this hyperstructure is a hypergroup but not a join space. I. Cristea, in [13], introduced conditions for the associated hypergroup to be a join space.

The notion of intuitionistic fuzzy sets was first introduced by Atanassov, in [4]. For details of intuitionistic fuzzy sets, we refer the reader to $[4,5]$.

This paper is structured as follows. After the introduction, in Section 2, were call some basic notions and results on hypergroups. In Section 3, we associate finite hyperstructures with fuzzy sets endowed with $n$-ary membership functions. In Section 4, we study the associated hyperstructures with hypergroups and join spaces. We prove that the new hyperstructure is a commutative hypergroup but, in general, it is not a join space. We then give some conditions such that the aforementioned commutative hypergroup also has this property. In Section 5, we study the set of all intuitionistic fuzzy sub-hypergroups of a hypergroup and give several examples and establish some characterization theorems.

It is worth mentioning that in this paper we deal only with finite hypergroupoids.

\section{Preliminaries}

We recall some definitions (see $[7,12]$ ), needed in what follows.

Let $H$ be a non-empty set and $P^{*}(H)$ be the family of all non-empty subsets of $H$. A hyperoperation or join operation is a map $\circ: H \times H \rightarrow P^{*}(H)$. For each pair $(a, b) \in H \times H$, we denote $\circ(a, b)$ by $a \circ b$.

The join operation is extended to subsets of $H$ in a natural way, namely

$$
A \circ B:=\bigcup\{a \circ b \mid a \in A, b \in B\} \text {. }
$$

The notations $a \circ A$ and $A \circ a$ are used for $\{a\} \circ A$ and $A \circ\{a\}$ respectively. Generally, the singleton $\{a\}$ is identified by its element $a$.

A non-empty set $H$, endowed with a hyperoperation "०" is called a hypergroupoid and it is denoted by $(H, \circ)$. If

$$
x \circ(y \circ z)=(x \circ y) \circ z, \forall x, y, z \in H,
$$

then $(H, \circ)$ is called a semihypergroup.

A hypergroupoid $(H, \circ)$ is called a quasihypergroup, if $a \circ H=H \circ a=H$, for all $a \in H$.

Definition 2.1. A hypergroup is a semihypergroup and a quasihypergroup.

Definition 2.2. Let $K \subseteq H, K \neq \phi$. We say that $(K, \circ)$ is a subhypergroup of $(H, \circ)$ if for any $a \in K$, we have $a \circ K=K \circ a=K$.

For $a, b \in H$, we denote: $a / b=\{x \mid a \in x \circ b\}$ and $b \backslash a=\{x \mid a \in b \circ x\}$.

Definition 2.3. A commutative hypergroup $(H, \circ)$ is called a join space if the following condition holds:

$$
a / b \cap c / d \neq \phi \Rightarrow a \circ d \cap b \circ c \neq \phi, \quad \forall a, b, c, d \in H .
$$

Join spaces were introduced by W. Prenowitz, in [16], and represent an important subclass of hypergroups with many applications especially in geometry (see [17]). 
Corsini, in [8], showed that to any hypergroupoid $H$ endowed with a fuzzy set, we can associate a join space $\left(H, \circ_{1}\right)$ as follows:

$$
\forall(x, y) \in H^{2}, \quad x \circ{ }_{1} y=\{z \in H \mid \mu(x) \wedge \mu(y) \leq \mu(z) \leq \mu(x) \vee \mu(y)\} .
$$

Recently, Corsini, in [10], has defined a new hypergroupoid associated with two membership functions. Here we recall this construction. Let $\mu, \lambda: H \rightarrow R^{+}$be two functions defined on a nonempty set $H$. For $x, y \in H$, we define the hyperproduct of $x, y$ with respect to $\mu$, $\lambda$ by

$$
\forall(x, y) \in H^{2}, x \circ_{\mu \lambda} y=\left\{z \in H \mid \begin{array}{l}
\mu(x) \wedge \mu(y) \wedge \lambda(x) \wedge \lambda(y) \leq \mu(z) \wedge \lambda(z) \\
\mu(x) \vee \mu(y) \vee \lambda(x) \vee \lambda(y) \geq \mu(z) \vee \lambda(z)
\end{array}\right\} .
$$

The obtained hyperstructure $\left(H,{ }_{\mu \lambda}\right)$ is a commutative hypergroup, but generally it is not a join space.

\section{3. $n$-ary membership functions}

A fuzzy set (of type 1) on a non-empty set $H$ is a function $\mu: H \rightarrow[0,1]$. The complement of $\mu$ denoted by $\mu^{c}$, is the fuzzy set of $H$ given by $\mu^{c}(x)=1-\mu(x)$ for all $x \in H$.

Let $\mu_{1}, \mu_{2}, \ldots, \mu_{n}: H \rightarrow[0,1]$, be $n$-ary fuzzy sets defined on a non-empty set $H$. For any $x, y \in H$, we can define a hyperstructure on $H$ by

$$
x \circ_{n} y=\left\{z \in H \mid \begin{array}{l}
\wedge_{i=1}^{n}\left(\mu_{i}(x) \wedge \mu_{i}(y)\right) \leq \wedge_{i=1}^{n} \mu_{i}(z) \\
\vee_{i=1}^{n}\left(\mu_{i}(x) \vee \mu_{i}(y)\right) \geq \vee_{i=1}^{n} \mu_{i}(z)
\end{array}\right\} .
$$

where $n$ is an element of the set $N^{*}$ of all non-zero natural numbers. For $a \in H$, we shall use the symbol $\wedge_{i=1}^{n} \mu_{i}(a)$ by $m(a)$ and $\vee_{i=1}^{n} \mu_{i}(a)$ by $M(a)$, for the sake of simplicity. Thus, we have

$$
x \circ_{n} y=\left\{z \in H \mid \begin{array}{l}
m(x) \wedge m(y) \leq m(z) \\
M(x) \vee M(y) \geq M(z)
\end{array}\right\} .
$$

By the definition of "o ${ }_{n}$ ", we have $\{x, y\} \subseteq x \circ_{n} y$.

Proof of the following proposition, which is omitted, is easy.

Proposition 3.1. Suppose $x, y \in H$, we define

This implies

$$
x \circ^{j} y=\left\{z \in H \mid \begin{array}{l}
\wedge_{i=n-j+1}^{n}\left(\mu_{i}(x) \wedge \mu_{i}(y)\right) \leq \wedge_{i=n-j+1}^{n} \mu_{i}(z) \\
\vee_{i=n-j+1}^{n}\left(\mu_{i}(x) \vee \mu_{i}(y)\right) \geq \vee_{i=n-j+1}^{n} \mu_{i}(z)
\end{array}\right\} .
$$

where $1 \leq j<n$.

$$
\left(x \circ_{n-j} y\right) \cap\left(x \circ^{j} y\right) \subseteq x \circ_{n} y,
$$

Remark. The set $\left(x \circ_{n-j} y\right) \cup\left(x \circ^{j} y\right)$ is not always a subset of $x \circ_{n} y$, as we can noticein the following example:

Example 3.2. Let $H=\{a, b, c, d\}$ and 


$$
\begin{array}{lll}
\mu_{1}(a)=0.15 & \mu_{2}(a)=0.40 & \mu_{3}(a)=0.25, \\
\mu_{1}(b)=0.45 & \mu_{2}(b)=0.50 & \mu_{3}(b)=0.70, \\
\mu_{1}(c)=0.45 & \mu_{2}(c)=0.60 & \mu_{3}(c)=0.90, \\
\mu_{1}(d)=0.20 & \mu_{2}(d)=0.35 & \mu_{3}(d)=0.20 .
\end{array}
$$

Then, we have

$$
a \circ_{3} b=\{a, b, d\}, \quad a \circ_{1} b=H, \quad a \circ^{2} b=\{a, b\} .
$$

This implies that

$$
\left(a \circ \circ_{1} b\right) \cap\left(a \circ^{2} b\right)=\{a, b\} \subset a \circ_{3} b=\{a, b, d\},
$$

$\operatorname{but}\left(a \circ \circ_{1} b\right) \cup\left(a \circ^{2} b\right)=H \supset a \circ_{3} b=\{a, b, d\}$.

Proposition 3.3. For all $x, y \in H$, we have

$$
\left(x \circ{ }_{n} x\right) \cup\left(y \circ{ }_{n} y\right) \subseteq x \circ_{n} y .
$$

Proof. Suppose $z \in X \circ_{n} x$, we have $m(x) \leq m(z)$ and $M(x) \geq M(z)$. For all $y \in H$, it implies that $m(x) \wedge m(y) \leq m(x) \leq m(z)$ and $M(x) \vee M(y) \geq M(x) \geq M(z)$, so $z \in x \circ_{n} y$, i.e., $x \circ_{n} x \subseteq x \circ_{n} y$. Similarly, one can deduce that $y \circ_{n} y \subseteq x \circ_{n} y$. Thus $\left(x \circ_{n} x\right) \cup\left(y \circ{ }_{n} y\right) \subseteq x \circ_{n} y$.

Theorem 3.4. Let

$$
\begin{aligned}
x \cdot{ }_{n} y & =\{z \in H \mid m(x) \leq m(z)\}, \\
x \bullet_{n} y & =\{z \in H \mid M(x) \geq M(z)\} .
\end{aligned}
$$

Then

$$
x \circ_{n} y=\left(\left(x \cdot{ }_{n} x\right) \cap\left(x \bullet \bullet_{n} x\right)\right) \cup\left(\left(y \cdot{ }_{n} y\right) \cap\left(y \bullet{ }_{n} y\right)\right) \cup\left(\left(x \cdot{ }_{n} x\right) \cap\left(y \bullet \bullet_{n} y\right)\right) \cup\left(\left(y \cdot{ }_{n} y\right) \cap\left(x \bullet{ }_{n} x\right)\right) .
$$

Proof. If we let $m(a)=\mu(a) \wedge v(a)$ and $M(a)=\mu(a) \vee v(a)$, for $a \in H$, then the proof will follow from Theorem 2 of [9].

\section{The relationship of hyperstructure $\left(H, \circ_{n}\right)$ and hypergroups and join spaces}

When $n=1$ and $n=2$ hypergroup $\left(H, \circ_{n}\right)$ has been studied by Corsini.

Let $n=1$. Then $m(a)=\mu_{1}(a)=M(a)$, for any $a \in H$, which means

$$
x \circ_{1} y=\{z \in H \mid \mu(x) \wedge \mu(y) \leq \mu(z) \leq \mu(x) \vee \mu(y)\},
$$

where $\mu_{1}(a)=\mu(a)$, for all $a \in H$.

Theorem 4.1. (See Corsini [9]). The hyperstructure $\left(H, \circ_{1}\right)$ is a join space.

Theorem 4.2. (See Corsini [10]). The hyperstructure $\left(H, \circ_{2}={ }_{\mu \lambda}\right)$ is a commutative hypergroup.

From now, we assume $n \geq 3$.

Theorem 4.3. The hyperstructure $\left(H, \circ_{n}\right)$ is a commutative hypergroup.

Proof. The hyperstructure $\left(H, \circ_{n}\right)$ is a quasihypergroup, because we have $\{x, y\} \subseteq x \circ_{n} y$ for all $x, y \in H$. On the other hand, suppose

$$
P(x, y, w)=\left\{z \in H \mid \begin{array}{l}
m(x) \wedge m(y) \wedge m(w) \leq m(z) \\
M(x) \vee M(y) \vee M(w) \geq M(z)
\end{array}\right\} .
$$

We prove that $\left(x \circ_{n} y\right) \circ_{n} w=P(x, y, w)$. We have 


$$
\left(x \circ_{n} y\right) \circ_{n} w=\left\{z \in H \mid \exists t:\left\{\begin{array}{l}
m(x) \wedge m(y) \leq m(t), m(t) \wedge m(w) \leq m(z) \\
M(t) \vee M(w) \geq M(z), M(x) \vee M(y) \geq M(t)
\end{array}\right\}\right\} .
$$

Let $z \in\left(x \circ_{n} y\right) \circ_{n} w$. Then we get

$$
\begin{aligned}
& m(x) \wedge m(y) \wedge m(w) \leq m(t) \wedge m(w) \leq m(z), \\
& M(x) \vee M(y) \vee M(w) \geq M(t) \vee M(w) \geq M(z) .
\end{aligned}
$$

This implies that $z \in P(x, y, w)$, and so

$$
\left(x \circ{ }_{n} y\right) \circ{ }_{n} w \subseteq P(x, y, w) .
$$

Conversely, suppose $z \in P(x, y, w)$, we have

$$
z \in\left(x \circ_{n} y\right) \circ_{n} w \Leftrightarrow \exists t: t \in X \circ_{n} y, \quad z \in t \circ_{n} w .
$$

Set

$$
\begin{aligned}
& m=m(x) \wedge m(y) \wedge m(w), \\
& M=M(x) \vee M(y) \vee M(w) .
\end{aligned}
$$

Assume $m=m(x), M=M(w)$ and $t=x$, then

$$
\begin{aligned}
& m=m(x)=m(t) \wedge m(w) \leq m(z), \\
& M=M(w)=M(t) \vee M(w) \geq M(z) .
\end{aligned}
$$

It implies $z \in t \circ_{n} w$. Since $t=x$, we have

$$
\begin{aligned}
& m(x) \wedge m(y) \leq m(t), \\
& M(x) \vee M(y) \geq M(t) .
\end{aligned}
$$

Thus $x=t \in x \circ_{n} y$, which means $z \in\left(x \circ_{n} y\right) \circ_{n} w$. One can deal with other cases by similar arguments. Hence

$$
\left(x \circ{ }_{n} y\right) \circ{ }_{n} w \supseteq P(x, y, w) .
$$

It follows that $\left(x \circ_{n} y\right) \circ_{n} w=P(x, y, w)$. Similarly, one gets $x \circ_{n}\left(y \circ_{n} w\right)=P(x, y, w)$. It implies that $\left(H, \circ_{n}\right)$ is a semihypergroup. Which means $\left(H, \circ_{n}\right)$ is a hypergroup.

Generally, $x \circ_{n} y$ is not a join space. Here, we give some examples of fuzzy sets $\mu_{1}, \mu_{2}, \ldots, \mu_{n}$ such that the associated hypergroup $\left(H, \circ_{n}\right)$ is a join space.

Example 4.4. Let $H=\{a, b, c, d, e\}$ and

$$
\begin{array}{lll}
\mu_{1}(a)=0.15 & \mu_{2}(a)=0.45 & \mu_{3}(a)=0.35, \\
\mu_{1}(b)=0.45 & \mu_{2}(b)=0.55 & \mu_{3}(b)=0.45, \\
\mu_{1}(c)=0.25 & \mu_{2}(c)=0.60 & \mu_{3}(c)=0.55, \\
\mu_{1}(d)=0.20 & \mu_{2}(d)=0.30 & \mu_{3}(d)=0.40, \\
\mu_{1}(e)=0.05 & \mu_{2}(e)=0.60 & \mu_{3}(e)=0.50 .
\end{array}
$$

Then, we have $m(a)=0.15, M(a)=0.45, m(b)=0.45, M(b)=0.55, m(c)=0.25, M(c)=0.60$, $m(d)=0.20, M(d)=0.40$, and $m(e)=0.05, M(e)=0.60$.

Consequently,

$$
a / b=\left\{x \in H \mid a \in x \circ_{3} b\right\}=\left\{z \in H \mid \begin{array}{l}
m(z) \wedge 0.45 \leq 0.15 \\
M(z) \vee 0.55 \geq 0.45
\end{array}\right\}=\{a, e\},
$$

and similarly $c / d=\{c, e\}$. On the other hand, we obtain

$$
a \circ_{3} d=\left\{z \in H \mid \begin{array}{l}
m(z) \geq 0.15 \\
M(z) \leq 0.45
\end{array}\right\}=\{a, d\}, \quad b \circ_{3} c=\left\{z \in H \mid \begin{array}{l}
m(z) \geq 0.25 \\
M(z) \leq 0.60
\end{array}\right\}=\{b, c\} .
$$


It implies that $e \in a / b \cap c / d \neq \phi$, but $\left(a \circ_{3} d\right) \cap\left(b \circ_{3} c\right)=\phi$. Hence $\left(H, \circ_{3}\right)$ is not a join space. For any $a \in H$, we denote:

$$
\begin{aligned}
& m_{i}(a)=\mu_{1}(a) \wedge \ldots \wedge \mu_{i-1}(a) \wedge \mu_{i+1}(a) \wedge \ldots \wedge \mu_{n}(a), \\
& M_{i}(a)=\mu_{1}(a) \vee \ldots \vee \mu_{i-1}(a) \vee \mu_{i+1}(a) \vee \ldots \vee \mu_{n}(a) .
\end{aligned}
$$

Theorem 4.5. For any $a \in H$, let there exist $i, j \in N^{*}$, such that

$$
m_{i}(a) \geq \mu_{i}(a), M_{j}(a) \leq \mu_{j}(a) \text { and } \mu_{i}=\mu_{j}^{c} .
$$

Then the hypergroup $\left(H, \circ_{n}\right)$ is a join space.

Proof. By our assumptions, the hyperoperation $" \circ_{n} "$ is given by

We used here the fact that

$$
x \circ_{n} y=\left\{z \in H \mid \begin{array}{l}
\mu_{i}(x) \wedge \mu_{i}(y) \leq \mu_{i}(z) \\
\mu_{j}(x) \vee \mu_{j}(y) \geq \mu_{j}(z)
\end{array}\right\} .
$$

$$
\mu_{i}(x) \wedge \mu_{i}(y) \leq \mu_{i}(z) \Leftrightarrow \mu_{j}(x) \vee \mu_{j}(y) \geq \mu_{j}(z),
$$

for any $x, y, z \in H$.

For Condition (III), suppose $\mu_{i}(x)=\mu_{i}(x) \wedge \mu_{i}(y) \leq \mu_{i}(z)$, then we have $\mu_{i}(x) \leq \mu_{i}(z)$ if and only if $\mu_{i}^{c}(x) \geq \mu_{i}^{c}(z)$. Since $\mu_{i}=\mu_{j}^{c}$, we have $\mu_{i}^{c}(x) \geq \mu_{i}^{c}(z)$ if and only if $\mu_{j}(x) \geq \mu_{j}(z)$.

On the other hand, we have

$$
\begin{aligned}
\mu_{i}(x)=\mu_{i}(x) \wedge \mu_{i}(y) & \Leftrightarrow \mu_{i}(x) \vee \mu_{i}(y)=\mu_{i}(y), \\
& \Leftrightarrow \mu_{i}^{c}(x) \vee \mu_{i}^{c}(y)=\mu_{i}^{c}(x), \\
& \Leftrightarrow \mu_{j}(x) \vee \mu_{j}(y)=\mu_{j}(x) .
\end{aligned}
$$

This implies $\mu_{j}(x) \geq \mu_{j}(z)$ if and only if $\mu_{j}(x) \vee \mu_{j}(y) \geq \mu_{j}(z)$.

Thus, we conclude that

$$
x \circ_{n} y=\left\{z \in H \mid \mu_{i}(x) \wedge \mu_{i}(y) \leq \mu_{i}(z)\right\} .
$$

Now, by Theorem 4 in [9], the hypergroup $\left(H, \circ_{n}\right)$ is a join space.

Example 4.6. Let $H=\{a, b, c, d, e\}$ and

$$
\begin{array}{llll}
\mu_{1}(a)=0.15 & \mu_{2}(a)=0.45 & \mu_{3}(a)=0.35 & \mu_{3}(a)=0.85, \\
\mu_{1}(b)=0.45 & \mu_{2}(b)=0.55 & \mu_{3}(b)=0.45 & \mu_{3}(b)=0.55, \\
\mu_{1}(c)=0.25 & \mu_{2}(c)=0.60 & \mu_{3}(c)=0.55 & \mu_{3}(c)=0.75, \\
\mu_{1}(d)=0.20 & \mu_{2}(d)=0.30 & \mu_{3}(d)=0.40 & \mu_{3}(d)=0.80, \\
\mu_{1}(e)=0.05 & \mu_{2}(e)=0.60 & \mu_{3}(e)=0.50 & \mu_{3}(e)=0.95 .
\end{array}
$$

In Example 4.4, we showed that $H=\{a, b, c, d, e\}$ with $\mu_{1}, \mu_{2}, \mu_{3}$ is not a join space. But since

$$
m_{1}(a) \geq \mu_{1}(a) \text { and } M_{4}(a) \leq \mu_{4}(a)
$$

for all $a \in H$ and $\mu_{1}=\mu_{4}^{c}$, by Theorem 4.5, we have $H=\{a, b, c, d, e\}$ with $\mu_{1}, \mu_{2}, \mu_{3}, \mu_{4}$ is a join space.

Theorem 4.7. For any $a \in H$, let there exist $i, j \in N^{*}$, such that

$$
m_{i}(a) \geq \mu_{i}(a), M_{j}(a) \leq \mu_{j}(a) \text { and } \mu_{i}=\mu_{j} .
$$

Then the hypergroup $\left(H, \circ_{n}\right)$ is a join space.

Proof. By our assumptions, we have 


$$
x \circ_{n} y=\left\{z \in H \mid \mu_{i}(x) \wedge \mu_{i}(y) \leq \mu_{i}(z) \leq \mu_{i}(x) \vee \mu_{i}(y)\right\} .
$$

Thus, the commutative hypergroup $\left(H, \circ_{n}\right)$ is a join space.

Theorem 4.8. For any $a \in H$, let there exist $i, j \in N^{*}$, such that

$$
\begin{aligned}
& m_{i}(a) \geq \mu_{i}(a) \text { and } M_{j}(a) \leq \mu_{j}(a)=\text { constant }, \\
& m_{i}(a) \geq \mu_{i}(a)=\text { constant and } M_{j}(a) \leq \mu_{j}(a) .
\end{aligned}
$$

Then the hypergroup $\left(H, \circ_{n}\right)$ is a join space.

Proof. By our assumptions imply that the hyperoperation "。 ${ }_{n}$ is given by

$$
x \circ_{n} y=\left\{z \in H \mid \mu_{i}(x) \wedge \mu_{i}(y) \leq \mu_{i}(z)\right\} \text {, }
$$

or

$$
x \circ_{n} y=\left\{z \in H \mid \mu_{j}(x) \wedge \mu_{j}(y) \geq \mu_{j}(z)\right\} .
$$

Thus, by Theorem 4 in [9], the hypergroup $\left(H, \circ_{n}\right)$ is a join space.

Theorem 4.9. (see Cristea [13]). Let $\mu$, $\lambda$ be two fuzzy sets in $H$ such that $\lambda=\mu^{c}$, for any $x \in H$. Then the commutative hypergroup $\left(H,{ }_{\mu \mu^{c}}\right)$ is a join space.

Lemma 4.10. Let $\mu_{1}, \mu_{2}, \ldots, \mu_{n}$ be $n$-ary fuzzy sets in $H$ such that $\mu_{i}=\mu_{n-i+1}^{c}$, for all $i, 1 \leq i \leq n$. Then, for $a, b, c, d \in H$, the following statements hold:

i) $\left(a \circ_{n} b\right) \cap\left(c \circ \circ_{n} d\right) \neq \phi$,

ii) $b / c \cap a / d \neq \phi$.

Proof.

i) Let $a, b, c, d \in H$. Since $\mu_{1}, \mu_{2}, \ldots, \mu_{n}$ are fuzzy sets in $H$, there exists an element $x \in H$ such that $m(x)=\sup _{h \in H} m(h)$, which implies that $M(x)=\inf _{h \in H} M(h)$. Since we have $\mu_{i}=\mu_{n-i+1}^{c}$, we get

$$
\begin{aligned}
& m(a) \wedge m(b) \leq m(x), \\
& M(a) \vee M(b) \geq M(x) .
\end{aligned}
$$

Therefore $x \in a \circ_{n} b$. Similarly, we can obtain $x \in c \circ_{n} d$. This implies that $\left(a \circ_{n} b\right) \cap\left(c \circ_{n} d\right) \neq \phi$.

ii) Similarly, there exists an element $y \in H$ such that $m(y)=\inf _{h \in H} m(h)$, which implies $M(y)=\sup _{h \in H} M(h)$. Thus, we have

$$
y \circ_{n} d=\left\{z \in H \mid \begin{array}{l}
m(d) \wedge m(y) \leq m(z) \\
M(d) \vee M(y) \geq M(z)
\end{array}\right\}=H .
$$

Which implies $y \in a / d$. Similarly, we have $y \in b / c$, and so $b / c \cap a / d \neq \phi$.

The following theorems are the main results of this section:

Theorem 4.11. By the same assumptions of Lemma 4.10, the commutative hypergroup $\left(H, \circ_{n}\right)$ is a join space.

Proof. By Lemma 4.10 (i), for any $a, b, c, d \in H$, we have

$$
b / c \cap a / d \neq \phi \Rightarrow\left(a \circ{ }_{n} b\right) \cap\left(c \circ_{n} d\right) \neq \phi,
$$

which means that $\left(H, \circ_{n}\right)$ is a join space.

Example 4.12. Back to Example 4.4, suppose $\mu_{4}=\mu_{3}^{c}, \mu_{5}=\mu_{2}^{c}, \mu_{6}=\mu_{1}^{c}$, then by Theorem 4.11, the set $H=\{a, b, c, d, e\}$ with $\mu_{1}, \mu_{2}, \mu_{3}, \mu_{4}, \mu_{5}, \mu_{6}$ is a join space. 
Theorem 4.13. Let $\mu=\mu_{1}, \mu_{2}, \ldots, \mu_{n-1}, \mu_{n}=\lambda$ be $n$-ary fuzzy sets in $H$. Let $\left(H,{ }_{\mu \lambda}\right)$ be a join space such that for all $x \in H$,

$$
\mu(x) \wedge \lambda(x) \leq \wedge_{i=2}^{n-1} \mu_{i}(x), \quad \mu(x) \vee \lambda(x) \geq \vee_{i=2}^{n-1} \mu_{i}(x) .
$$

Then the hypergroup $\left(H, \circ_{n}\right)$ is a join space.

Proof. It is enough to show that $x{ }_{\mu \lambda} y=x \circ_{n} y$ for all $x, y \in H$. By our assumptions, we get $\mu(x) \wedge \lambda(x)=m(x)$ and $\mu(x) \vee \lambda(x)=M(x)$, for all $x \in H$. Thus, we have $z \in x \circ{ }_{\mu \lambda} y$ if and only if

$$
\begin{gathered}
m(x) \wedge m(y)=\mu(x) \wedge \lambda(x) \wedge \mu(y) \wedge \lambda(y) \leq \mu(z) \wedge \lambda(z)=m(z), \\
M(x) \vee M(y)=\mu(x) \vee \lambda(x) \vee \mu(y) \vee \lambda(y) \geq \mu(z) \vee \lambda(z)=M(z),
\end{gathered}
$$

if and only if $z \in x \circ_{n} y$. This completes the proof.

By Theorem 4.13 and Theorem 4.9 we have the following results:

Corollary 4.14. Let $\mu=\mu_{1}, \mu_{2}, \ldots, \mu_{n-1}, \mu_{n}=\lambda$ be $n$-ary fuzzy sets in $H$ such that $\mu=\lambda^{c}$, and assume

$$
\mu(x) \wedge \lambda(x) \leq \wedge_{i=2}^{n-1} \mu_{i}(x), \quad \mu(x) \vee \lambda(x) \geq \vee_{i=2}^{n-1} \mu_{i}(x),
$$

for all $x \in H$. Then the hypergroup $\left(H, \circ_{n}\right)$ is a join space.

Corollary 4.15. Let $\mu=\mu_{1} \leq \mu_{2} \leq \ldots \leq \mu_{n}=\lambda$ be $n$-ary fuzzy sets in $H$ such that $\mu=\lambda^{c}$. Then the hypergroup $\left(H, \circ_{n}\right)$ is a join space. Here $\mu_{i} \leq \mu_{j}$, means $\mu_{i}(a) \leq \mu_{j}(a)$, for all $x \in H$.

\section{Intuitionistic fuzzy sets of the hypergroup $\left(H, \circ_{n}\right)$}

The notion of intuitionistic fuzzy sets was first introduced by Atanassov in [4]. For details of intuitionistic fuzzy sets, we refer the reader to [4, 5]. In [6], Biswas applied the concept of intuitionistic fuzzy sets to the theory of groups and studied intuitionistic fuzzy subgroups of a group. Dudek et al., in [14], considered the intuitionistic fuzzification of the concept of subhyperquasigroups in a hyperquasigroup and investigated some properties of such hyperquasigroups.

In this section, we apply the concept of intuitionistic fuzzy sets to the hypergroup $\left(H, \circ_{n}\right)$ and we investigate some related properties.

Definition 5.1. An intuitionistic fuzzy set $A$ in a non-empty set $X$ is an object having the form

$$
A=\left\{\left(x, \mu_{A}(x), \lambda_{A}(x)\right) \mid x \in X\right\},
$$

where the functions $\mu_{A}: X \rightarrow[0,1]$ and $\lambda_{A}: X \rightarrow[0,1]$ denote the degree of membership (namely $\mu_{A}(x)$ ) and the degree of nonmembership (namely $\lambda_{A}(x)$ ) of each element $x \in X$ with respect to the set $A$, respectively, and $0 \leq \mu_{A}(x)+\lambda_{A}(x) \leq 1$ for all $x \in X$.

For the sake of simplicity, we shall use the symbol $A=\left(\mu_{A}, \lambda_{A}\right)$ for the intuitionistic fuzzy set $A=\left\{\left(x, \mu_{A}(x), \lambda_{A}(x)\right) \mid x \in X\right\}$. Denote by $\operatorname{IF}(X)$ the set of all intuitionistic fuzzy sets in $X$.

Definition 5.2. Let $(H, \circ)$ be a hypergroup (resp. hyperquasigroup). An intuitionistic fuzzy set $A=\left(\mu_{A}, \lambda_{A}\right)$ in $H$ is called an intuitionistic fuzzy sub-hypergroup (resp. sub-hyperquasigroup) of $(H, \circ)$ if the following axioms hold:

(1) $\mu_{A}(x) \wedge \mu_{A}(y) \leq \inf _{z \in x \circ y} \mu_{A}(z)$ for all $x, y \in H$;

(2) for all $x, a \in H$ there exist $y, z \in H$ such that $x \in(a \circ y) \cap(z \circ a)$ and

$$
\mu_{A}(a) \wedge \mu_{A}(x) \leq \mu_{A}(y) \wedge \mu_{A}(z)
$$


(3) $\lambda_{A}(x) \vee \lambda_{A}(y) \geq \sup _{z \in x \circ y} \lambda_{A}(z)$ for all $x, y \in H$;

(4) for all $x, a \in H$ there exist $y, z \in H$ such that $x \in(a \circ y) \cap(z \circ a)$ and

$$
\lambda_{A}(y) \vee \lambda_{A}(z) \leq \lambda_{A}(a) \vee \lambda_{A}(x) \text {. }
$$

For the sake of simplicity, we shall use the symbol IFSH for the intuitionistic fuzzy sub-hypergroup. Here we suppose $A=H$ and denote $\lambda_{X}=\lambda, \mu_{X}=\mu$ and also, let $\mu_{1}, \mu_{2}, \ldots, \mu_{n}$ be $n$-ary fuzzy sets in $H$.

Corollary 5.3. For all $x \in H$, let $0 \leq \wedge_{i=1}^{n} \mu_{i}(x)+\vee_{i=1}^{n} \mu_{i}(x) \leq 1$. Then

$$
\left(\wedge_{i=1}^{n} \mu_{i}(x), \vee_{i=1}^{n} \mu_{i}(x)\right)
$$

is always a IFSH of $\left(H,{ }_{n}\right)$.

Proof. Since the hyperstructure $\left(H, \circ_{n}\right)$ is a commutative hypergroup such that $\{x, y\} \subseteq x \circ_{n} y$, for any $x, y \in H$, thus, the conditions (2) and (4) of Definition 5.2, are verified. On the other hand, for all $x, y, z \in H$ such that $z \in X \circ y$, by definitions we have

$$
\left(\wedge_{i=1}^{n} \mu_{i}(x)\right) \wedge\left(\wedge_{i=1}^{n} \mu_{i}(y)\right) \leq \wedge_{i=1}^{n} \mu_{i}(z) \text { and }\left(\vee_{i=1}^{n} \mu_{i}(x)\right) \vee\left(\vee_{i=1}^{n} \mu_{i}(y)\right) \geq \vee_{i=1}^{n} \mu_{i}(z) .
$$

Hence, the conditions (1) and (3) of Definition 5.2 are satisfied.

Remark. $\left(\vee_{i=1}^{n} \mu_{i}, \wedge_{i=1}^{n} \mu_{i}\right),\left(\vee_{i=1}^{n} \mu_{i}, \vee_{i=1}^{n} \mu_{i}\right)$ and $\left(\wedge_{i=1}^{n} \mu_{i}, \wedge_{i=1}^{n} \mu_{i}\right)$ are not always a IFSH of $\left(H, \circ_{n}\right)$, as we can notice below.

Example 5.4. In Example 4.4, we have $a \in e \circ_{3} b$. On the other hand, we have $\vee_{i=1}^{3} \mu_{i}(e)=0.60$ and $\vee_{i=1}^{3} \mu_{i}(b)=0.55$. Thus

$$
\left(\vee_{i=1}^{3} \mu_{i}(e)\right) \wedge\left(\vee_{i=1}^{3} \mu_{i}(b)\right)=0.55>0.45=\vee_{i=1}^{3} \mu_{i}(a) .
$$

This means the condition (1) of Definition 5.2 is not satisfied.

Similarly, we have $c \in e \circ_{3} d$, but

$$
\left(\wedge_{i=1}^{3} \mu_{i}(e)\right) \vee\left(\wedge_{i=1}^{3} \mu_{i}(d)\right)=0.20>0.25=\wedge_{i=1}^{3} \mu_{i}(c) .
$$

This means the condition (3) of Definition 5.2 is not satisfied.

Lemma 5.5. $\left(\vee_{i=1}^{n} \mu_{i}(x)\right)^{c}=\wedge_{i=1}^{n} \mu_{i}^{c}(x)$ for all $x \in H$.

Proof. For all $x \in H$ there exists $s \in\{1, \ldots, n\}$ such that $\vee_{i=1}^{n} \mu_{i}(x)=\mu_{s}(x)$. Hence, for all $i \in\{1, \ldots, n\}$ we have $\mu_{i}(x) \leq \mu_{s}(x)$. This implies that $\mu_{i}^{c}(x) \geq \mu_{s}^{c}(x)$. Thus $\wedge_{i=1}^{n} \mu_{i}^{c}(x) \geq \mu_{s}^{c}(x)$.

On the other hand, obvious that $\wedge_{i=1}^{n} \mu_{i}^{c}(x) \leq \mu_{s}^{c}(x)$. This implies $\wedge_{i=1}^{n} \mu_{i}^{c}(x)=\mu_{s}^{c}(x)=\left(\vee_{i=1}^{n} \mu_{i}(x)\right)^{c}$.

Lemma 5.6. $\left(\wedge_{i=1}^{n} \mu_{i}(x)\right)^{c}=\vee_{i=1}^{n} \mu_{i}^{c}(x)$ for all $x \in H$.

Theorem 5.7. For all $x \in H$, let $\vee_{i=1}^{n} \mu_{i}(x)=\frac{1}{2}$. Then $\left(\vee_{i=1}^{n} \mu_{i}, \vee_{i=1}^{n} \mu_{i}\right)$ is a IFSH of $\left(H, \circ_{n}\right)$ if and only if $\left(\wedge_{i=1}^{n} \mu_{i}^{c}, \wedge_{i=1}^{n} \mu_{i}^{c}\right)$ is a IFSH of $\left(H, \circ_{n}\right)$.

Proof.

$\Rightarrow$ Since $\vee_{i=1}^{n} \mu_{i}(x)=\frac{1}{2}$ for all $x \in H$, then we have $\wedge_{i=1}^{n} \mu_{i}^{c}(x)=\frac{1}{2}$, thus $\left(\wedge_{i=1}^{n} \mu_{i}^{c}, \wedge_{i=1}^{n} \mu_{i}^{c}\right)$ is a $I F$ of $\left(H, \circ_{n}\right)$. Let $\left(\vee_{i=1}^{n} \mu_{i}, \vee_{i=1}^{n} \mu_{i}\right)$ be a IFSH of $\left(H, \circ_{n}\right)$. Then for all $x, y \in H$, we have

$$
\left(\vee_{i=1}^{n} \mu_{i}(x)\right) \wedge\left(\vee_{i=1}^{n} \mu_{i}(y)\right) \leq \inf _{z \in X \circ y}\left\{\vee_{i=1}^{n} \mu_{i}(z)\right\},
$$

and so 


$$
\left[1-\left(\vee_{i=1}^{n} \mu_{i}(x)\right)^{c}\right] \wedge\left[1-\left(\vee_{i=1}^{n} \mu_{i}(y)\right)^{c}\right] \leq \inf _{z \in x \circ y}\left\{1-\left(\vee_{i=1}^{n} \mu_{i}(z)\right)^{c}\right\}
$$

which implies

$$
\left[1-\left(\vee_{i=1}^{n} \mu_{i}(x)\right)^{c}\right] \wedge\left[1-\left(\vee_{i=1}^{n} \mu_{i}(y)\right)^{c}\right] \leq 1-\sup _{z \in \times \circ y}\left\{\left(\vee_{i=1}^{n} \mu_{i}(z)\right)^{c}\right\}
$$

thus

Therefore

$$
\sup _{z \in x \circ y}\left\{\left(\vee_{i=1}^{n} \mu_{i}(z)\right)^{c}\right\} \leq 1-\left(\left[1-\left(\vee_{i=1}^{n} \mu_{i}(x)\right)^{c}\right] \wedge\left[1-\left(\vee_{i=1}^{n} \mu_{i}(y)\right)^{c}\right]\right),
$$

By lemma 5.5, we have

$$
\sup _{z \in x \circ y}\left\{\left(\vee_{i=1}^{n} \mu_{i}(z)\right)^{c}\right\} \leq\left(\vee_{i=1}^{n} \mu_{i}(x)\right)^{c} \vee\left(\vee_{i=1}^{n} \mu_{i}(y)\right)^{c},
$$

$$
\sup _{z \in x \circ y}\left\{\wedge_{i=1}^{n} \mu_{i}^{c}(z)\right\} \leq\left(\wedge_{i=1}^{n} \mu_{i}^{c}(x)\right) \vee\left(\wedge_{i=1}^{n} \mu_{i}^{c}(y)\right)
$$

hence the third condition of Definition 5.2 is verified. Now, let $x, a \in H$. Then thereexist $y, z \in H$ such that $x \in(a \circ y) \cap(z \circ a)$ and

$$
\left(\vee_{i=1}^{n} \mu_{i}(a)\right) \wedge\left(\vee_{i=1}^{n} \mu_{i}(x)\right) \leq\left(\vee_{i=1}^{n} \mu_{i}(y)\right) \wedge\left(\vee_{i=1}^{n} \mu_{i}(z)\right),
$$

This implies

$$
\left[1-\left(\vee_{i=1}^{n} \mu_{i}(a)\right)^{c}\right] \wedge\left[1-\left(\vee_{i=1}^{n} \mu_{i}(x)\right)^{c}\right] \leq\left[1-\left(\vee_{i=1}^{n} \mu_{i}(y)\right)^{c}\right] \wedge\left[1-\left(\vee_{i=1}^{n} \mu_{i}(z)\right)^{c}\right],
$$

Hence

$$
\left(\vee_{i=1}^{n} \mu_{i}(a)\right)^{c} \vee\left(\vee_{i=1}^{n} \mu_{i}(x)\right)^{c} \geq\left(\vee_{i=1}^{n} \mu_{i}(y)\right)^{c} \vee\left(\vee_{i=1}^{n} \mu_{i}(z)\right)^{c},
$$

by lemma 5.5, we have

$$
\left(\wedge_{i=1}^{n} \mu_{i}^{c}(a)\right) \vee\left(\wedge_{i=1}^{n} \mu_{i}^{c}(x)\right) \geq\left(\wedge_{i=1}^{n} \mu_{i}^{c}(y)\right) \vee\left(\wedge_{i=1}^{n} \mu_{i}^{c}(z)\right),
$$

and the fourth condition of Definition 5.2 is satisfied. Similarly, the conditions (1) and (2) of Definition 5.2 are verified.

$\Leftarrow)$ The proof is similar to that of above.

Theorem 5.8. For all $x \in H$, let $\wedge_{i=1}^{n} \mu_{i}(x)=\frac{1}{2}$. Then $\left(\wedge_{i=1}^{n} \mu_{i}, \wedge_{i=1}^{n} \mu_{i}\right)$ is a IFSH of $\left(H, \circ_{n}\right)$ if and only if $\left(\vee_{i=1}^{n} \mu_{i}^{c}, \vee_{i=1}^{n} \mu_{i}^{c}\right)$ is a IFSH of $\left(H, \circ_{n}\right)$.

Proof. The proof is similar to that of Theorem 5.7.

Corollary 5.9. For any $a \in H$, let there exist $i, j \in N^{*}$, such that

$$
m_{i}(a) \geq \mu_{i}(a), M_{j}(a) \leq \mu_{j}(a) \text { and } \mu_{i}=\mu_{j}^{c} .
$$

Then $\left(\mu_{i}, \mu_{i}^{c}\right)$ is a IFSH of $\left(H,{ }_{n}\right)$.

Proof. The statement of this Theorem follows immediately from Theorem 4.5 and the proof of Theorem 5.7.

Corollary 5.10. For any $a \in H$, let there exist $i, j \in N^{*}$, such that

$$
m_{i}(a) \geq \mu_{i}(a), M_{j}(a) \leq \mu_{j}(a) \text { and } \mu_{i}=\mu_{j} .
$$

Then $\left(\mu_{i}, \mu_{i}^{c}\right)$ and $\left(\mu_{i}^{c}, \mu_{i}\right)$ are a IFSH of $\left(H, \circ_{n}\right)$.

Proof. The latter proof would be implied by Theorem 4.7 and the proof of Theorem 5.7.

Corollary 5.11. For any $a \in H$, let there exist $i, j \in N^{*}$, such that

$$
m_{i}(a) \geq \mu_{i}(a) \text { and } M_{i}(a) \leq \mu_{i}(a)=\text { constant. }
$$

Then $\left(\mu_{j}, \mu_{j}^{c}\right)$ is a IFSH of $\left(H, \circ_{n}\right)$.

Proof. The statement of this Theorem follows immediately from Theorem 4.8 and the proof of Theorem 5.7.

Similarly, by Theorem 4.8 and the proof of Theorem 5.7, we find the next result: 
M. Asghari-Larimi, P. Corsini, E. Ranjbar-Yanehsari / TJMCS Vol .5 No. 2 (2012) 115-125

Corollary 5.12. For any $a \in H$, let there exist $i, j \in N^{*}$, such that

$$
m_{i}(a) \geq \mu_{i}(a)=\text { constant and } M_{i}(a) \leq \mu_{i}(a) \text {. }
$$

Then $\left(\mu_{j}^{c}, \mu_{j}\right)$ is a IFSH of $\left(H, \circ_{n}\right)$.

Acknowledgement. The author is highly grateful to the referees for their constructive suggestions for improving the paper.

\section{References.}

[1] M. Asghari-Larimi, Some properties of intuitionistic nil radicals of intuitionistic fuzzy ideals, International Mathematical Forum, 5 (2010), 1551 - 1558.

[2] M. Asghari-Larimi and B. Davvaz, Hyperstructures associated to arithmetic functions, Ars Combitoria, 97 (2010), 51-63.

[3] M. Asghari-Larimi and V. Leoreanu-Fotea, A connection between hypergroupoids and L-Fuzzy Sets of Type 2, Italian J. of Pure and Appl. Math., 26 (2009), 207-216.

[4] K.T. Atanassov, Intuitionistic fuzzy sets, Fuzzy Sets Syst., 20 (1986), 87-96.

[5] K.T. Atanassov, New operations defined over the intuitionistic fuzzy sets, Fuzzy Sets Syst., 61 (1994), 137-142.

[6] R. Biswas, Intuitionistic fuzzy subgroups, Math. Forum, 10 (1989), 37-46.

[7] P. Corsini, Prolegomena of Hypergroup Theory, Aviani Editore, 1993.

[8] P. Corsini, Join Spaces, Power Sets, Fuzzy Sets, Proceedings of the 5th A.H.A. Congress, 1993, Iasi (Romania) Hadronic Press, (1994), 45-52.

[9] P. Corsini, Hyperstructures associated with ordered sets, Bull. Greek Math. Soc., 48 (2003), 7-18. [10] P. Corsini, Hyperstructures associated with fuzzy sets endowed with two membership functions, j. of combin. infor. system sci., 1-4 (2006), 247-254.

[11] P. Corsini, A new connection between hypergroups and fuzzy sets, Southeast Asian Bull. Math., 27 (2003), 221-229.

[12] P. Corsini and V. Leoreanu-Fotea, Applications of Hyperstructure Theory, Kluwer Academic Publications, Dordrecht, Advances in Mathematics, 2003.

[13] I. Cristea, Hyperstructures and fuzzy sets endowed with two membership functions, Fuzzy sets and Systems, 160 (2009), 1114-1124.

[14] W.A. Dudek, B. Davvaz and Y.B. Jun, On intuitionistic fuzzy sub-quasihypergroups of quasihypergroups, Information Sciences, 170 (2005), 251-262.

[15] F. Marty, Sur une generalisation de la notion de groupe, 8th course Math. Scandinaves Stockholm, (1934), 45-49.

[16] W. Prenowitz, Projectives Geometries as Multigroups, Amer. J. Math., 65 (1943), 235-256.

[17] W. Prenowitz and J. Jantosciak, Join geometries, Springer-Verlag, UTM, 1979.

[18] T. Vougiouklis, Hyperstructures and their representations, Hadronic Press, Inc, 115, PalmHarber, USA, 1994.

[19] L.A. Zadeh, Fuzzy Sets, Inform and Control, 8 (1965), 338-353. 